\title{
U-shaped neck pillow for chondrodermatitis nodularis helicis: A budget- friendly, simple alternative
}

\author{
Arooj Mohammed, BS; Michael Isaacs, MD; Sahand Rahnama, MD, MS \\ Department of Dermatology, Indiana University School of Medicine, Indianapolis, Indiana
}

Key words: budget-friendly; chondrodermatitis nodularis helicis; $\mathrm{CNH}$; convenient; management; pillow Abbreviation used: $\mathrm{CNH}$ (chondrodermatitis nodularis helicis)

\section{Therapeutic challenge}

Chondrodermatitis nodularis helicis $(\mathrm{CNH})$ results from inflammation of the cartilage of the ear, specifically of the helix or antihelix. $\mathrm{CNH}$ most commonly occurs because the patient sleeps on the affected side and may also be due to sun exposure in men, who more commonly drive and are consequently vulnerable to light on the left-hand side. $\mathrm{CNH}$ frequently recurs unless the patient avoids repeated trauma by reducing pressure applied over the area affected. Methods suggested in the literature include attachment of ear-protective devices, sleeping with a custom $\mathrm{CNH}$ pillow or hemorrhoid pillow, or advising the patient to alter his/her sleeping position. However, some of our patients have found difficulty finding an effective $\mathrm{CNH}$ or hemorrhoid pillow.

\section{Solution}

A solution suggested by one of our patients that we have adopted is using a U-shaped neck pillow, a lowcost, readily portable and accessible, pressure-relieving device most frequently bought by consumers for relief during long-distance car rides or flights. Despite being devised to support the neck and back during travel, neck pillows can also provide a cavity through which the cartilage can recover overnight (Fig 1). The patient is advised to purchase such a cushion on which he/she can rest the head during sleep, allowing the ear to recover in the hole. U-shaped pillows are commercially available in many convenience stores and online, and they are adaptable to use, easily transportable, and less expensive than many other interventions. We advocate their use as a long-lasting, budget-friendly solution for CNH in affected patients.

Funding sources: None.

Conflicts of interest: None disclosed. 
Fig 1. Example of a U-shaped neck pillow, which can be used to relieve pressure on ear while supporting the head and neck (U-Shape Memory Foam Travel Pillow; Modern Sleep, Jessup, MD). (Image reproduced with permission of Classic Brands Specialty Sleep Products.)

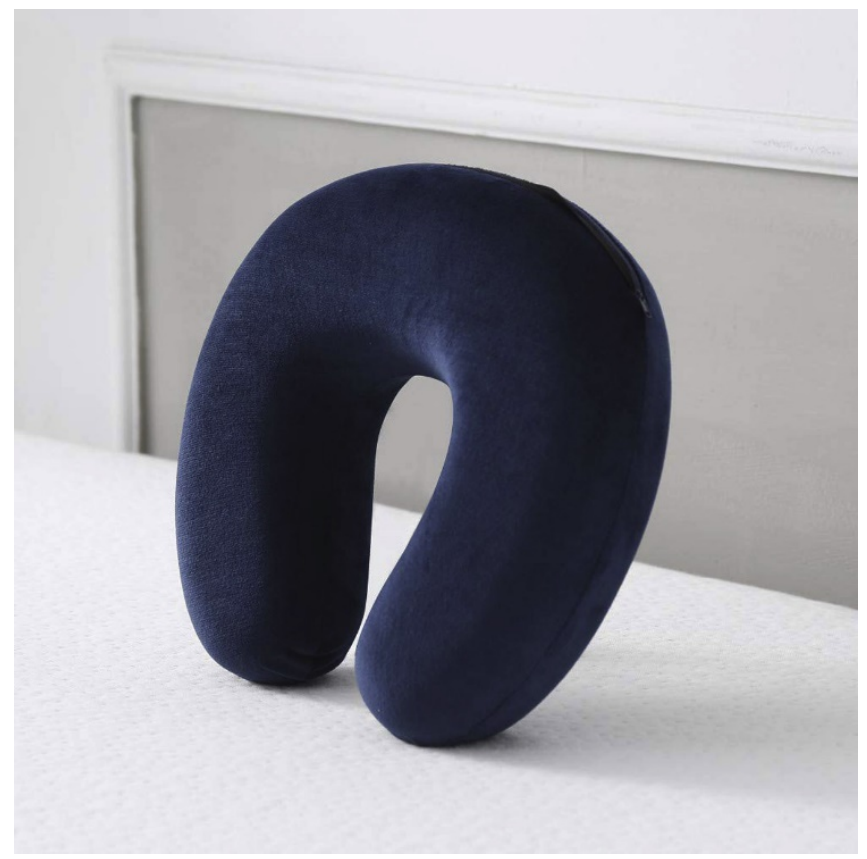

